e-Journal of Educational

Research, Assessment and Evaluation

\section{RELIEVE}

Revista ELectrónica de Investigación y EValuación Educativa

\title{
LA VOZ DEL ESTUDIANTE EN LA EVALUACIÓN DEL APRENDIZAJE: UN CAMINO POR RECORRER EN LA UNIVERSIDAD
}

\section{[Student voice in learning assessment: a pathway not yet developed at university]}

by/por

Article record

$\underline{\text { About authors }}$

HTML format

\author{
Rodríguez-Gómez, Gregorio (gregorio.rodriguez@uca.es) \\ Ibarra-Sáiz, Maria Soledad (marisol.ibarra@uca.es) \\ Gallego-Noche, Beatriz (beatriz.gallego@uca.es) \\ Gómez-Ruiz, Miguel-Ángel (miguel.gomez@uca.es) \\ Quesada-Serra, Victoria (victoria.quesada@uca.es)
}

$\underline{\text { Ficha del artículo }}$

$\underline{\text { Sobre los autores }}$

Formato HTML

\begin{abstract}
During 2009/2010, the current research was conducted with the aim of analysing student and teaching staff on regarding student participation in assessment. A content analysis of 76 subject outlines was carried out, and then 40 member of the teaching staff and 614 university students were surveyed. The results of the content analysis prove there is a shortage of information about and programming for student participation in assessment.
\end{abstract}

\section{Keywords}

Learning assessment, Learning-oriented assessment, Selfassessment, Peer-assessment, Co-assessment, Collaborative assessment, Participative assessment, Higher Education.

\section{Resumen}

Durante el curso 2009/2010 se llevó a cabo esta investigación con el objetivo de analizar la opinión y perspectiva que profesores y estudiantes universitarios tienen sobre la participación de estos últimos en el proceso de evaluación. Se realizó un análisis de contenido de 76 programas de asignaturas universitarias y se encuestaron mediante dos cuestionarios a 40 profesores y 614 estudiantes universitarios. Los resultados muestran una escasez de evidencias sobre la participación real de los estudiantes. Además, confirman opiniones divergentes entre docentes y estudiantes sobre los usos y las formas en las que se concreta esta participación activa en la evaluación.

\section{Descriptores}

Evaluación del aprendizaje, evaluación orientada al aprendizaje, autoevaluación, evaluación entre iguales, coevaluación, evaluación colaborativa, evaluación participativa, educación superior.
Las recientes investigaciones en el contexto del aprendizaje universitario insisten en la necesidad de desarrollar en los estudiantes la capacidad para regular sus propios procesos de aprendizaje a través de la participación activa en los procedimientos de evaluación
(Boud, 2006; Boud \& Associates, 2010; Nicol, 2009). De hecho, desde la década de los ochenta, y sobre todo en la década de los noventa del pasado siglo, son numerosas las investigaciones que rompen con una tradición en la que el profesor es el actor princi- 
pal y único en la evaluación del aprendizaje. Estas investigaciones vienen a demostrar y reclamar la importancia de la participación activa de los estudiantes en los procesos de evaluación, siendo de destacar en este sentido la revisiones llevadas a cabo por parte de Falchikov (1986; 2005), Dochy, Segers y Sluijmans (1999) y la de Gielen, Docky y Onghena (2011).

Paralelamente, los cambios que se vienen produciendo a nivel socio-económico y cultural hacen que se exija a las instituciones de educación superior que los graduados sean capaces de planificar y mantener el seguimiento de su propio proceso de aprendizaje en etapas posteriores, desarrollando así las competencias del aprendizaje autónomo a lo largo de la vida y del pensamiento crítico e innovador, entre otras (Goñi, 2005).

En este contexto de innovación y cambio, el profesorado universitario está enfrentándose al reto de hacer partícipe al estudiante del proceso de enseñanza, a través de estrategias metodológicas participativas como el aprendizaje colaborativo o el aprendizaje basado en problemas. Pero es preciso también que el profesorado diseñe, planifique y desarrolle procedimientos de evaluación en los que juegue un papel central la implicación y participación activa de los estudiantes, teniendo en cuenta los beneficios que supone esta participación en el desarrollo del aprendizaje del alumnado.

Estas consideraciones previas exigen, tanto del profesorado como de los estudiantes, e incluso de la propia institución universitaria, una nueva cultura de la evaluación en la que la actividad evaluativa llegue a ser por sí misma tarea de aprendizaje. Tareas de evaluación/aprendizaje "auténticas" que, como señala Ibarra (1999), posibiliten la construcción del conocimiento, la investigación disciplinada y la transferencia del saber a otros contextos. Estas tareas auténticas son uno de los ejes centrales de la concepción evaluativa conocida como "evaluación orientada al aprendizaje", planteada por Carless, Joughin y Mok (2006), donde se considera la participación activa del alumnado universitario en el proceso de evaluación principalmente a través de estrategias como la autoevaluación, la evaluación entre iguales o la coevaluación; junto con una continua interacción docenteestudiante a través de un proceso de retroalimentación y proalimentación que permita la mejora del desempeño del estudiante. En definitiva, el rol del estudiante se amplía con el rol de evaluador. Del papel tradicional de carácter pasivo asignado a los estudiantes, según el cual los estudiantes son objeto de evaluación, se pasa a un enfoque activo en el que el estudiante es un agente evaluador.

Como manifiesta Boud (2006), la sociedad actual demanda algo diferente a simples graduados pasivos que se conforman con un régimen de evaluación predeterminado. Se pretende, en cambio, graduados que sean capaces de planificar y mantener un seguimiento de su propio proceso de aprendizaje de forma autónoma. En este contexto, se considera la participación de los estudiantes en la actividad evaluadora como una oportunidad de aprendizaje que, por sí misma, puede desarrollar competencias como:

- Pensamiento reflexivo, crítico e independiente (Sambell y McDowell, 1998; Sivan, 2000).

- Conocimiento a partir de valorar diferentes soluciones a diferentes problemas (Gibbs, 1981; 2003).

- Autosuficiencia y dirección del propio aprendizaje (Boud, 1991; 2000; Stefani, 1994).

- Debate, discusión y negociación (Prins y otros, 2005).

- Aprendizaje autónomo y autoconfianza (Brew, 2003).

En definitiva, mediante la educación en la evaluación se puede aprender a establecer criterios (y con ellos prioridades), a reflexionar sobre lo positivo y lo negativo de las realidades, a valorar (y comparar) los objetos 
de evaluación y sobre todo, a tomar decisiones fundamentadas y justificadas. De esta forma se puede favorecer que el estudiante autorregule su aprendizaje y fomentar su aprendizaje autónomo y, desde una perspectiva profesional, que se adapte más fácilmente a los cambios y esté capacitado para asumir responsabilidades.

Así pues, los avances científico-técnicos y los desarrollos en el campo del aprendizaje y la evaluación obligan a la educación universitaria a plantearse nuevas estrategias en la evaluación del aprendizaje que tengan en cuenta esta implicación activa del estudiante. Así son cada vez más los autores que vienen impulsando estrategias alternativas a la evaluación tradicional que ponen el énfasis en esta participación (Biggs, 2005; Biggs y Tang, 2009; Bordás y Cabrera, 2001; Boud, 2011; Boud \& Associates, 2010; Carless, Joughin y Mok, 2006; Falchikov, 2005; Gessa, 2011; Gibbs, 2006; Ibarra Sáiz y Rodríguez Gómez, 2010; Ibarra Sáiz, Rodríguez Gómez y Gómez Ruiz, 2012; Knight, 2005; Ljungman y Silén, 2008; López Pastor, 2009; Padilla Carmona y Gil Flores, 2008; Pérez Pueyo y otros, 2008; Rodríguez Gómez e Ibarra Sáiz, 2011; Rodríguez Gómez, Ibarra Sáiz y Gómez Ruiz, 2011).

La investigación que se presenta forma parte de un estudio más amplio ${ }^{[1]}$ a través del cual se pretendía desarrollar procedimientos e instrumentos de evaluación que facilitaran y promovieran la participación de los estudiantes en el proceso evaluativo. Los beneficios de este empeño redundarían en la calidad de la enseñanza que ofrece la universidad, en el mayor desarrollo de competencias académico-profesionales y humanas de los profesionales egresados y en la mutua satisfacción del profesorado y el alumnado con la oferta educativa de la enseñanza universitaria.

Como primer paso para conseguir esta finalidad se planteó identificar y describir la situación de partida en cada una de las uni- versidades participantes en el estudio, en relación con la participación de sus estudiantes en los procesos de evaluación del aprendizaje, a través de:

- Análisis documental de las estrategias que favorecen la participación del alumnado en el proceso de evaluación del aprendizaje y que están recogidas y especificadas en los programas de las asignaturas.

- Análisis mediante encuesta de las opiniones y percepciones, de docentes y estudiantes, sobre las estrategias evaluativas puestas en práctica en las asignaturas que pudieran favorecer la participación de los estudiantes en el proceso de evaluación de su aprendizaje.

\section{Método}

El diseño global utilizado en la investigación fue el de un "estudio de casos múltiple" (Rodríguez Gómez, Gil Flores y García Jiménez, 1999: 96), ya que los objetivos de la investigación eran fundamentalmente de tipo descriptivo/evaluativos, para lo que se utilizaron cinco casos, correspondiendo cada uno de ellos a una universidad participante en el proyecto. En este estudio se presentan exclusivamente los datos y resultados correspondientes al caso específico de la Universidad de Cádiz, concretado en un análisis documental de los programas de las asignaturas y un proceso de encuesta a profesorado y estudiantes.

\section{Muestra}

\section{Población y muestra del análisis documental}

Durante el curso 2009/2010 en la Universidad de Cádiz se impartieron un total de 65 titulaciones oficiales (de ciclo corto, de ciclo largo, de sólo segundo ciclo y másteres). Para esta investigación se procedió al análisis de los programas docentes de materias de las cinco ramas de conocimiento. Los programas docentes, según normativa interna de la universidad (Instrucción UCA/I01VPOA/2010 de 20/12/2009), con el fin de coordinar los Planes de Ordenación Docente de Centros y 
Departamentos para el curso siguiente, deben ser aprobados y publicados en la página web institucional en el mes de junio del curso anterior.

El método de selección de programas para su revisión fue de carácter no probabilístico, concretamente se utilizó un muestreo por cuotas, en el que se determinó como criterio disponer de un mínimo de 75 programas en total. En la tabla 1 se presenta la composición final de la muestra de los documentos seleccionados para su análisis.

Tabla 1. Composición de la muestra de programas analizados por ramas de conocimiento

\begin{tabular}{|c|c|c|}
\hline Ramas de Conocimiento & $\mathbf{f}$ & $\%$ \\
\hline Artes y Humanidades & 16 & 21,1 \\
\hline Ciencias & 15 & 19,7 \\
\hline Ciencias de la Salud & 15 & 19,7 \\
\hline Ciencias Sociales y Jurídicas & 16 & 21,1 \\
\hline Ingeniería/Arquitectura & 14 & 18,4 \\
\hline Total & 76 & 100 \\
\hline
\end{tabular}

\section{Población y muestra de las encuestas}

La población de docentes de la Universidad de Cádiz, según la memoria académica del curso académico 2009/2010, estaba formada en ese momento por 1.541 profesores, organizados en torno a 48 departamentos universitarios. Utilizando el muestreo por cuotas, combinado con un muestreo incidental por cada una de las cinco áreas de conocimiento, se procedió a seleccionar al profesorado según criterios de facilidad de acceso $\mathrm{y}$ aceptación a participar. De acuerdo con este proceso de selección se encuestaron un total de 40 docentes (ver Tabla 2).

Tabla 2. Composición de la muestra de docentes encuestados por ramas de conocimiento

\begin{tabular}{|c|c|c|}
\hline Ramas de Conocimiento & f & $\%$ \\
\hline Artes y Humanidades & 5 & 12,5 \\
\hline Ciencias & 6 & 15 \\
\hline Ciencias de la Salud & 9 & 22,5 \\
\hline Ciencias Sociales y Jurídicas & 12 & 30 \\
\hline Ingeniería/Arquitectura & 8 & 20 \\
\hline Total & 40 & 100 \\
\hline
\end{tabular}

Respecto a la población de estudiantes, el total de alumnado matriculado, según la memoria del curso académico 2009/2010, ascendía a 17.280 estudiantes. Para la selección de la muestra se utilizó un procedimiento similar al del profesorado, combinando el muestreo por cuotas con el incidental. Se pudo tener acceso a los estudiantes a través de su profesorado, de acuerdo con criterios de facilidad de acceso y aceptación a participar quedando formada la muestra final productora de datos por un total de 614 estudiantes pertenecientes a diferentes ramas de conocimiento (ver Tabla 3).

TABLA 3. Composición de la muestra de estudiantes encuestados

\begin{tabular}{|c|c|c|}
\hline Ramas de Conocimiento & $\mathbf{f}$ & $\%$ \\
\hline Arte y Humanidades & 61 & 9,9 \\
\hline Ciencias & 62 & 10,1 \\
\hline Ciencias de la Salud & 154 & 25,1 \\
\hline Ciencias Sociales y Jurídicas & 184 & 29,9 \\
\hline Ingeniería/Arquitectura & 153 & 24,9 \\
\hline Total & 614 & 100 \\
\hline
\end{tabular}

\section{Instrumentos}

De acuerdo a la naturaleza de la información que se pretendía recabar se diseñaron dos tipos de instrumentos:

- Escala para el análisis documental de la participación de los estudiantes en el proceso de evaluación.

- Cuestionario sobre participación de los estudiantes en su evaluación, con dos versiones dirigidas a profesorado y estudiantes.

En cuanto a la validez de los instrumentos utilizados se llevó a cabo un proceso de validación de contenido por jueces expertos de cada una de las universidades participantes en el proyecto. Por otra parte los cálculos de fiabilidad realizados dan unos índices de fiabilidad (alfa de Cronbach) entre 0,75 y 0,78 . 
Escala para el análisis documental de la participación de los estudiantes en el proceso de evaluación

Para el análisis de los programas de las asignaturas se diseñó la "Escala para el análisis documental de la participación de los estudiantes en el proceso de evaluación". Este instrumento estaba compuesto por quince ítems o atributos clasificados en cinco dimensiones:

- Participación de los estudiantes en la evaluación.

- Diseño de la evaluación (criterios, métodos, instrumentos, etc.).

- Negociación entre estudiantes y docentes sobre distintos elementos de la evaluación.

- Retroalimentación y proalimentación.

- Modalidades de participación de los estudiantes -autoevaluación, evaluación entre iguales y/o coevaluación.

Para cada uno de los quince atributos se especificaba, en formato de lista de control (Sí/No), si aparecía o no alguna evidencia sobre cada uno de ellos. En caso afirmativo, se valoraba el grado en que se manifestaba el atributo a través de una escala de frecuencia con los siguientes valores: 1 (muy poco), 2 (poco), 3 (algo), 4 (bastante), 5 (mucho) o 6 (totalmente).

\section{Cuestionario sobre participación de los es-} tudiantes en su evaluación

Para recabar la opinión de estudiantes y docentes se elaboró un cuestionario con dos versiones: a) Cuestionario sobre participación de los estudiantes en su evaluación (versión para docentes); y b) Cuestionario sobre participación de los estudiantes en su evaluación (versión para estudiantes). Ambos instrumentos tenían un formato similar y se estructuraron en unas preguntas iniciales de identificación y en 21 cuestiones referidas a las actitudes y creencias del profesorado o de los estudiantes, según la versión, sobre la participación activa de los estudiantes en la evaluación. Las dimensiones en las que estaban estructurados estos instrumentos eran:

- Criterios de evaluación.

- Diseño de la evaluación.

- Información y formación/entrenamiento.

- Autoevaluación.

- Evaluación entre iguales y coevaluación.

- Opiniones sobre la participación de los estudiantes en la evaluación.

- Consecuencias y ponderación de la participación de los estudiantes en la evaluación en sus calificaciones.

Para cada una de las cuestiones, atendiendo a la naturaleza de la pregunta, la opinión se podía expresar en diferentes escalas, desde una lista de control indicado la presencia o ausencia (Sí/No) hasta una valoración mediante escala tipo Likert con valores comprendidos entre un mínimo de 1 y un máximo de 6 .

\section{Procedimiento}

\section{Análisis de los programas docentes}

El análisis de los programas de las asignaturas se centró únicamente en el apartado referido a la evaluación, apartado en el cual el profesorado debe especificar los criterios y procedimientos de la evaluación. A través de este análisis, se identificaron los aspectos planificados por el profesorado que estaban relacionados con la participación de los estudiantes en el proceso de evaluación.

Cada programa se analizó con la Escala para el análisis documental de la participación de los estudiantes en el proceso de evaluación. En primer lugar, se comprobaba si había alguna evidencia de la explicitación de cada uno de los quince atributos contemplados en la escala, determinando así la presencia o ausencia de la información referida a cada atributo. En segundo lugar, si se detectaba alguna evidencia referida a la participación de los estudiantes en el proceso de evaluación, se procedía a valorar en qué grado se explicitaba o manifestaba esta presencia. 
La información recogida con la Escala para el análisis documental de la participación de los estudiantes en el proceso de evaluación era mayoritariamente de carácter cuantitativo por lo que se realizó un estudio descriptivo, atendiendo a frecuencias y porcentajes de respuesta a las distintas opciones.

Para facilitar la interpretación de los datos y la presentación de resultados se optó por agrupar las valoraciones realizadas en tres categorías. Una primera categoría (nada) que hacía referencia a cuando no se encontraron evidencias de ese atributo en el programa. Si había evidencias en la planificación de la participación de los estudiantes en la evaluación, las valoraciones se agruparon en dos categorías: "algo" para las comprendidas entre los valores 1, 2 y 3; y "mucho" para las valoraciones 4,5 y 6 (ver Tabla 4).

\section{Análisis de las opiniones de profesorado y estudiantes}

La cumplimentación de los cuestionarios para docentes y estudiantes podía realizarse de forma "on-line" y también de forma tradicional en papel. En el caso de la muestra de docentes, todos los participantes utilizaron la versión "on-line" del instrumento por ser éste el medio más cómodo y rápido. Sin embargo, en el caso de los estudiantes, se consideró que el contexto de aula era el más idóneo para acceder a un mayor número de participantes, por lo que todos respondieron a la encuesta presencialmente.

Respecto al análisis de los datos, y teniendo en cuenta que la información que se obtuvo era mayoritariamente de carácter cuantitativo, se llevó a cabo un análisis estadístico de los datos mediante un estudio descriptivo (frecuencias y porcentajes) y pruebas de contraste no paramétricas. Las preguntas abiertas fueron contestadas sólo por parte de un número muy reducido de encuestados, por lo que sólo han sido utilizadas en la presentación de resultados cuando añaden un matiz relevante a la información proporcionada por el análisis estadístico.

\section{Resultados}

\section{La participación de los estudiantes en los programas docentes}

En un primer momento se presentan los resultados obtenidos en el análisis documental de los programas docentes (ver Tabla 4 y Figura 1). En este sentido, se constata que en los programas de las asignaturas existe muy poca información respecto a la planificación de participación de los estudiantes en la evaluación. Así, podemos observar cómo en más del $80 \%$ de los programas no aparece información relacionada con los ítems 14, 7 y 5:

- Favorecer la supervisión y entrenamiento de los estudiantes en procedimientos de evaluación.

- Proponer que los estudiantes participen en el diseño y construcción de instrumentos de evaluación.

- Acordar o consensuar con los estudiantes los criterios de evaluación.

Similarmente, en más del $50 \%$ de los programas de las asignaturas no aparece evidencia alguna referida a los atributos $2,3,6,8$, $10,11,12,13$ y 15 .

En casi un $20 \%$ de los programas se evidencia que los criterios de evaluación propician en alguna medida la participación de los estudiantes (ítem 4). Asimismo, en torno a un $15 \%$ de los programas especifica con claridad el peso de la participación de los estudiantes en la calificación (ítem 15), y se aporta información sobre los resultados de la evaluación de forma que los estudiantes puedan reflexionar sobre su nivel de logro (ítem 9). 
Rodríguez-Gómez, Gregorio; Ibarra, Marisol; Gallego-Noche, Beatriz; Gómez-Ruiz, Miguel-Ángel \& Quesada-Serra, Victoria (2012). La voz del estudiante en la evaluación del aprendizaje: un camino por recorrer en la universidad.

RELIEVE, v. 18, n. 2, art. 2. DOI: 10.7203/relieve.18.2.1985

Tabla 4. Grado de presencia de cada atributo en los programas de las asignaturas (\%)

\begin{tabular}{|c|c|c|c|}
\hline $\begin{array}{l}\text { Atributos } \\
\end{array}$ & Nada & Algo & Mucho \\
\hline $\begin{array}{l}\text { 1. Se especifican los beneficios de la participación de los estudiantes en el proceso de eva- } \\
\text { luación de los aprendizajes }\end{array}$ & 42,1 & 44,7 & 13,2 \\
\hline $\begin{array}{l}\text { 2. Se favorece la participación de los estudiantes en el diseño del proceso de evaluación } \\
\text { (diálogos de evaluación) }\end{array}$ & 57,9 & 32,9 & 9,2 \\
\hline $\begin{array}{l}\text { 3. Se propone acordar y/o consensuar conjuntamente con los estudiantes qué será objeto de } \\
\text { evaluación (determinar qué se va a evaluar: comunicación oral, aprendizaje autónomo, } \\
\text { conocimiento de conceptos básicos, etc.) }\end{array}$ & 71,1 & 22,4 & 6,6 \\
\hline $\begin{array}{l}\text { 4. Los criterios de evaluación propician la participación de los estudiantes en el proceso de } \\
\text { evaluación }\end{array}$ & 40,8 & 39,5 & 19,7 \\
\hline $\begin{array}{l}\text { 5. Se propone acordar y/o consensuar conjuntamente con los estudiantes los criterios de } \\
\text { evaluación (claridad expositiva, relevancia y adecuación de las actividades realizadas autó- } \\
\text { nomamente, precisión terminológica, etc.) }\end{array}$ & 80,3 & 18,4 & 1,3 \\
\hline $\begin{array}{l}\text { 6. Se propone acordar y/o consensuar conjuntamente con los estudiantes los instrumentos } \\
\text { de evaluación }\end{array}$ & 72,4 & 23,7 & 3,9 \\
\hline $\begin{array}{l}\text { 7. Se propone que los alumnos participen en el diseño y construcción de los instrumentos } \\
\text { de evaluación }\end{array}$ & 84,2 & 14,5 & 1,3 \\
\hline 8. Se propone acordar y/o consensuar con los estudiantes el sistema de calificación & 68,4 & 26,3 & 5,3 \\
\hline $\begin{array}{l}\text { 9. Se especifica/propone aportar a los estudiantes información sobre los resultados de la } \\
\text { evaluación de forma que puedan reflexionar sobre su nivel de logro (retroalimentación) }\end{array}$ & 40,8 & 44,7 & 14,5 \\
\hline $\begin{array}{l}\text { 10. Se especifica/propone aportar a los estudiantes información sobre los resultados de la } \\
\text { evaluación de forma que puedan modificar y mejorar sus ejecuciones (proalimentación) }\end{array}$ & 51,3 & 35,5 & 13,2 \\
\hline $\begin{array}{l}\text { 11. Se especifica/favorece la participación de los estudiantes a través de la autoevaluación } \\
\text { (evaluaciones del estudiante/grupo sobre sus actividades y ejecuciones) }\end{array}$ & 51,3 & 39,5 & 9,2 \\
\hline $\begin{array}{l}\text { 12. Se especifica/favorece la participación de los estudiantes a través de la evaluación entre } \\
\text { iguales (evaluaciones de los estudiantes/grupos de las actividades y ejecuciones de sus } \\
\text { compañeros) }\end{array}$ & 64,5 & 26,3 & 9,2 \\
\hline $\begin{array}{l}\text { 13. Se especifica/favorece la participación de los estudiantes a través de la coevaluación } \\
\text { (profesor y estudiantes evalúan de forma consensuada y negociada) }\end{array}$ & 68,4 & 23,7 & 7,9 \\
\hline $\begin{array}{l}\text { 14. Se especifica/favorece la supervisión y entrenamiento por otros estudiantes en procedi- } \\
\text { mientos de evaluación }\end{array}$ & 94,7 & 3,9 & 1,4 \\
\hline $\begin{array}{l}\text { 15. Se especifica el peso de la participación de los estudiantes en el proceso de evaluación } \\
\text { en la calificación final de la asignatura }\end{array}$ & 53,9 & 30,3 & 15,8 \\
\hline
\end{tabular}

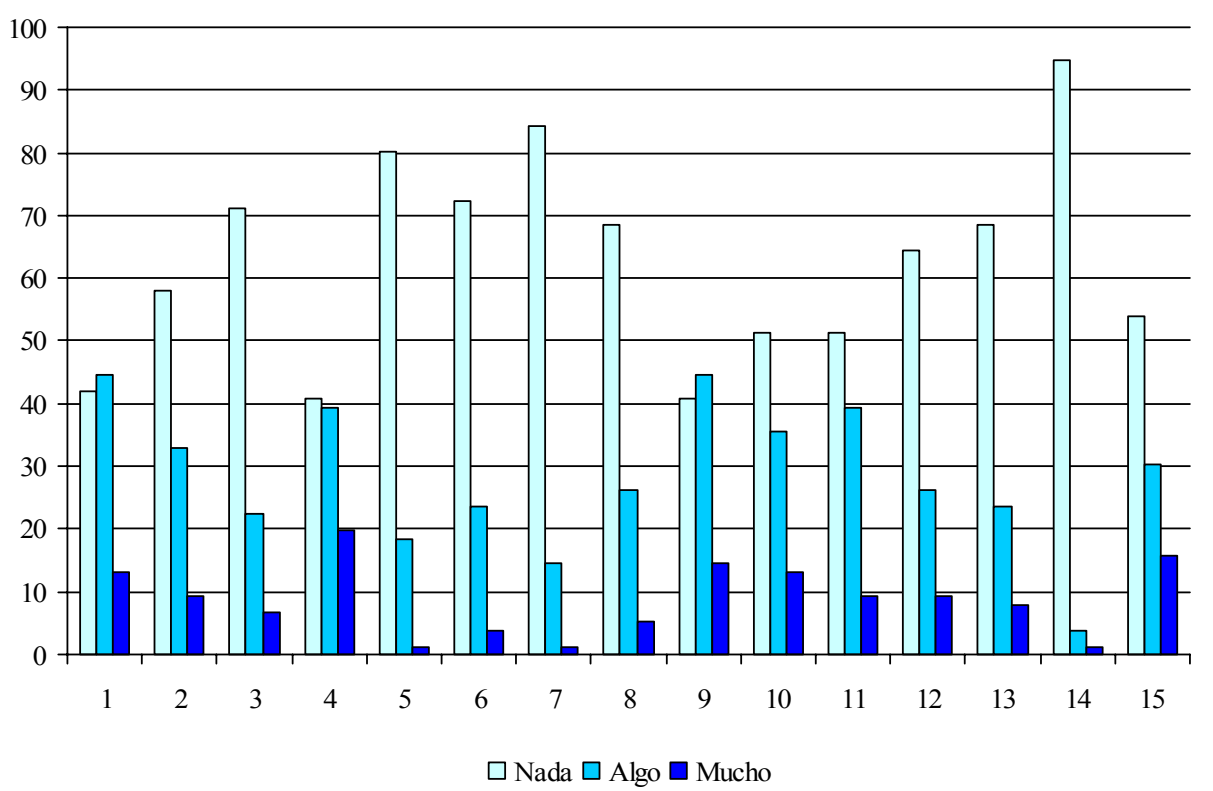

Figura 1. Grado de presencia por atributo (\%) 


\section{La participación de los estudiantes desde la perspectiva de profesorado y estu- diantes}

En este apartado se presentan los resultados obtenidos en las encuestas realizadas al profesorado y a los estudiantes. Se han agrupado según las dimensiones del estudio y se presentan en las tablas correspondientes los porcentajes o puntuaciones medias, según cada situación, destacándose mediante asterisco $(*)$, cuando corresponde, las diferencias estadísticamente significativas con una $\mathrm{p} \leq$ 0,05 .

\section{Criterios, diseño e información y entrena- miento/formación en evaluación}

Se hace referencia a los resultados de las tres primeras dimensiones del cuestionario: a) criterios de evaluación (del ítem 6.1 al 6.5); b) diseño de la evaluación (del ítem 7.1 al 7.5); y c) información y entrenamiento/formación (ítems 8, 9, 10, 11 y 12).

En la Figura 2 se presenta el porcentaje de profesores y estudiantes que responden afirmativamente a cada una de las cuestiones planteadas relativas a la participación de los estudiantes en la elaboración de los criterios de evaluación. La mayor diferencia la encontramos en las respuestas al ítem 6.1, ya que un $45 \%$ del profesorado señala como ade- cuado que los estudiantes participen en la determinación de criterios de evaluación, frente a un $88,6 \%$ de los estudiantes que afirman que es necesario.

Los porcentajes de respuesta afirmativa al ítem 6.2 tanto del alumnado como del profesorado son casi idénticos, siendo una minoría la que afirma que se ofrecen estrategias y espacios a los estudiantes para participar directamente en la planificación de la evaluación.

Tan sólo cinco de los cuarenta docentes encuestados (12,8\%) afirman que definen de forma conjunta con sus estudiantes los procedimientos de evaluación en sus asignaturas (ítem 6.3), siendo aún menor esta percepción desde el punto de vista de los estudiantes $(6,1 \%)$.

Es también destacable que el 72,5\% del profesorado afirma realizar actividades para explicar y discutir los criterios de evaluación (ítem 6.4); sin embargo, sólo lo percibe el $41,9 \%$ de los estudiantes. Después de esta discusión conjunta, el 47,5\% del profesorado afirma modificar los criterios de evaluación incorporando alguna propuesta de sus estudiantes (ítem 6.5). No obstante, sólo el $11,5 \%$ de los estudiantes reconoce que esa modificación tiene lugar.

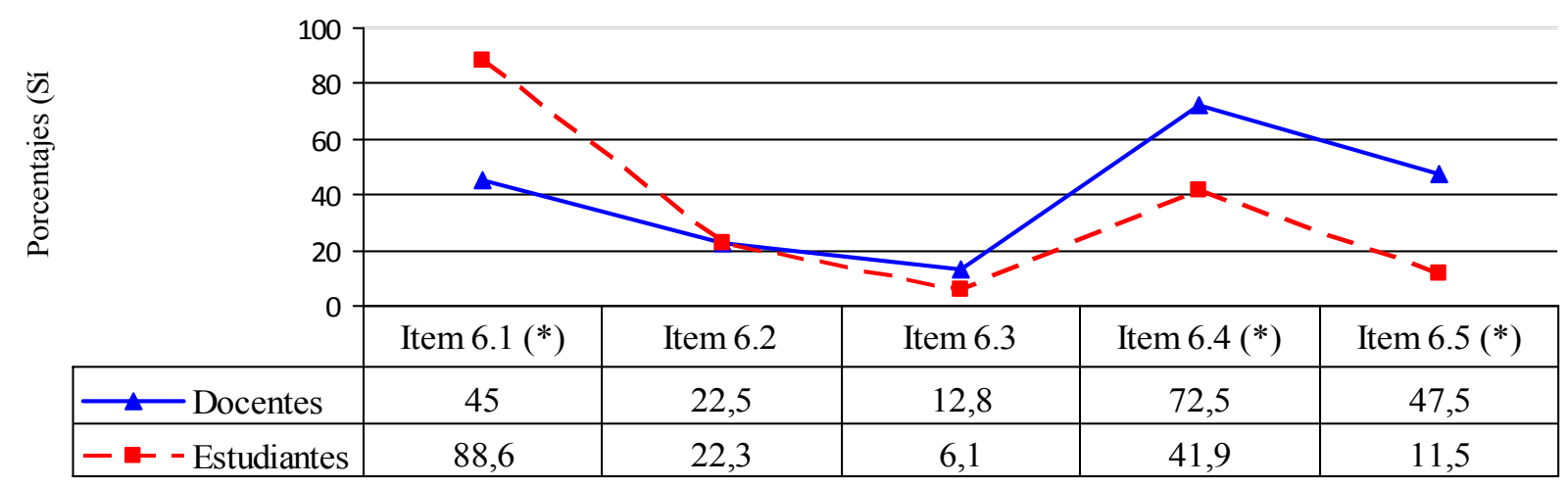

FIGURA 2. Perspectiva de profesores y estudiantes sobre la participación en el establecimiento de los criterios de evaluación

En cuanto a la participación del alumnado en el diseño de evaluación, la Figura 3 mues- tra que se ofrecen escasas posibilidades a los estudiantes para participar en el diseño del 
proceso de evaluación, pues los porcentajes de respuesta positiva a los ítems de esta di- mensión son todos bajos.

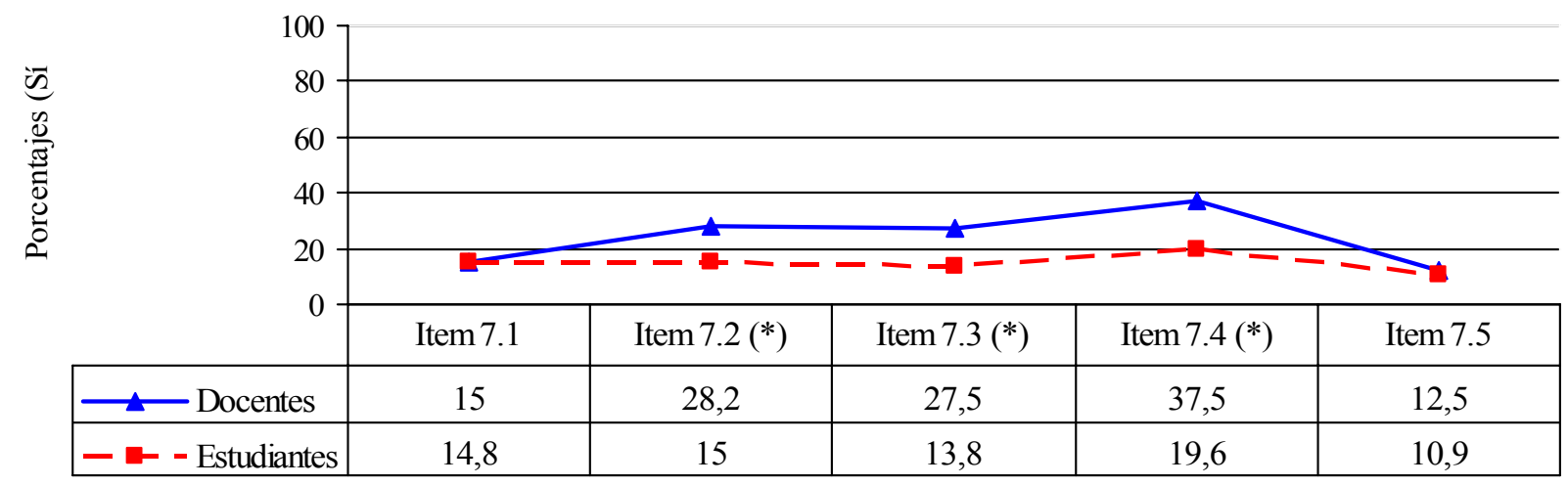

Figura 3. Perspectiva de profesores y estudiantes sobre la participación en el diseño de la evaluación

En cambio, hay en torno a un tercio del profesorado $(37,5 \%)$ que afirma ofrecer la posibilidad de elección de tareas para ser evaluadas (ítem 7.4). Tanto profesorado como estudiantes coinciden en señalar la baja participación del alumnado en la delimitación de lo que será objeto de evaluación -los contenidos teóricos, las tareas a realizar, entregables- (ítem 7.1) y en el establecimiento del procedimiento de calificación (ítem 7.5). La participación de los estudiantes en la elección de los instrumentos (ítem 7.2) y en la construcción/elaboración de los mismos (ítem 7.3) es percibida por los docentes en mayor medida, con un $28,2 \%$ y $27,5 \%$, respectivamente, que por el alumnado $(15 \% \mathrm{y}$ $13,8 \%)$.

En la dimensión sobre información y entrenamiento (Figura 4), se denota una cierta discrepancia en la opinión de profesores y estudiantes, siendo el porcentaje de respuestas afirmativas de los docentes a los ítems de esta dimensión en todos los casos bastante mayor que el de los estudiantes.
El 61,5\% de los docentes afirma ofrecer información a los estudiantes sobre los beneficios de participar en la evaluación (ítem 8); sin embargo, frente a esta opinión sólo el $36,1 \%$ de los estudiantes considera que esto sea así. Destacamos el hecho de que el 95\% del profesorado afirme que las estrategias que utilizan permiten al estudiante conocer su nivel de logro (ítem 10) y que proporcionan retroalimentación (ítem 11). Percepción que difiere de la manifestada por los estudiantes $(62,5 \%$ en el ítem 10 y $58,5 \%$ en el ítem 11). Mientras el $82,1 \%$ del profesorado declara proporcionar a sus estudiantes proalimentación o retroalimentación prospectiva (ítem 12), sólo el 32\% de los estudiantes afirma percibir ésta.

Es revelador el porcentaje de respuestas al ítem 9 sobre la presencia de actividades para entrenarse en la evaluación. Tanto para el alumnado ( $20 \%$ de respuestas afirmativas) como para el profesorado $(38,5 \%)$ representa el porcentaje más bajo en los ítems de esta dimensión. 


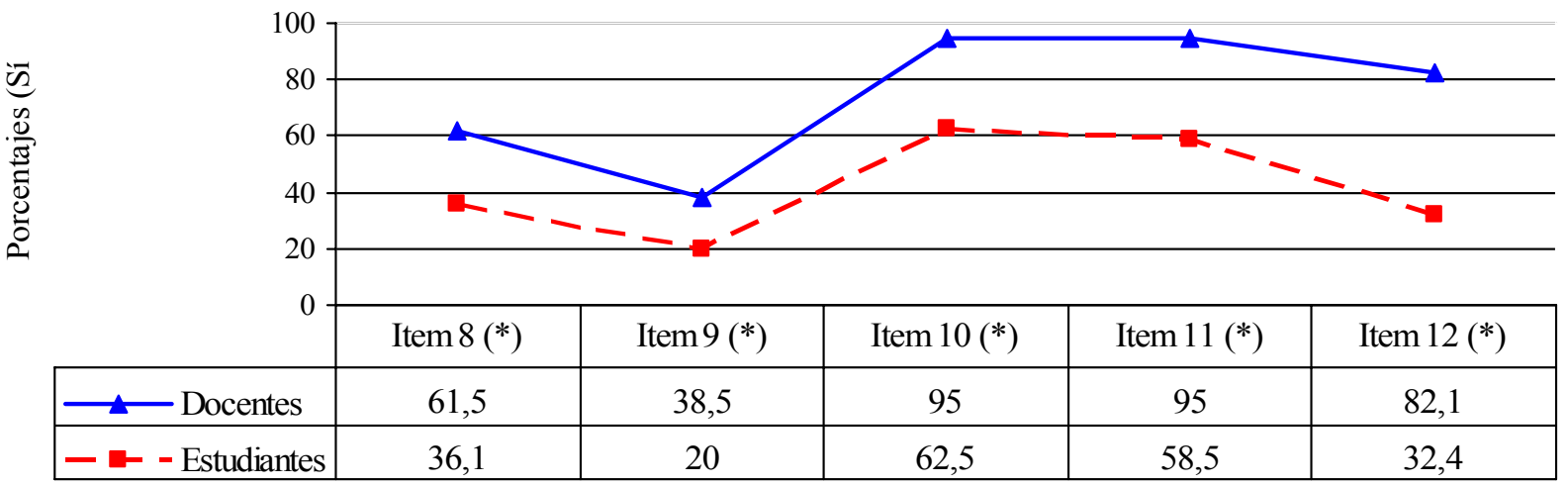

Figura 4. Perspectiva de profesores y estudiantes sobre la información aportada en las prácticas evaluativas

Modalidades de evaluación: autoevaluación, evaluación entre iguales y coevaluación

La encuesta para la recogida de información incluía varios ítems para conocer la opinión de los docentes y los estudiantes sobre el uso y modo de utilización de la autoevaluación, la evaluación entre iguales y la coevaluación.

En primer lugar, la autoevaluación se reflejaba en dos ítems (13 y 14). En el caso del ítem 13 podemos observar en la Figura 5 cómo se presentan dos perfiles con tendencias de respuesta bastante similares entre profesores y estudiantes. Para el $17,3 \%$ de los estudiantes y el $30 \%$ del profesorado la autoevaluación se pone en práctica a través de la valoración de ejecuciones individuales (ítem 13.1); o bien a través de la valoración de ejecuciones de grupo (ítem 13.2), opinión mantenida por el $9 \%$ de los estudiantes y el $12,5 \%$ del profesorado. Casi un tercio del profesorado (ítem 13.3) manifiesta que utiliza ambas modalidades de autoevaluación (individual o grupal), aunque sólo se muestran de acuerdo con esta afirmación el 15,4\% de los estudiantes.

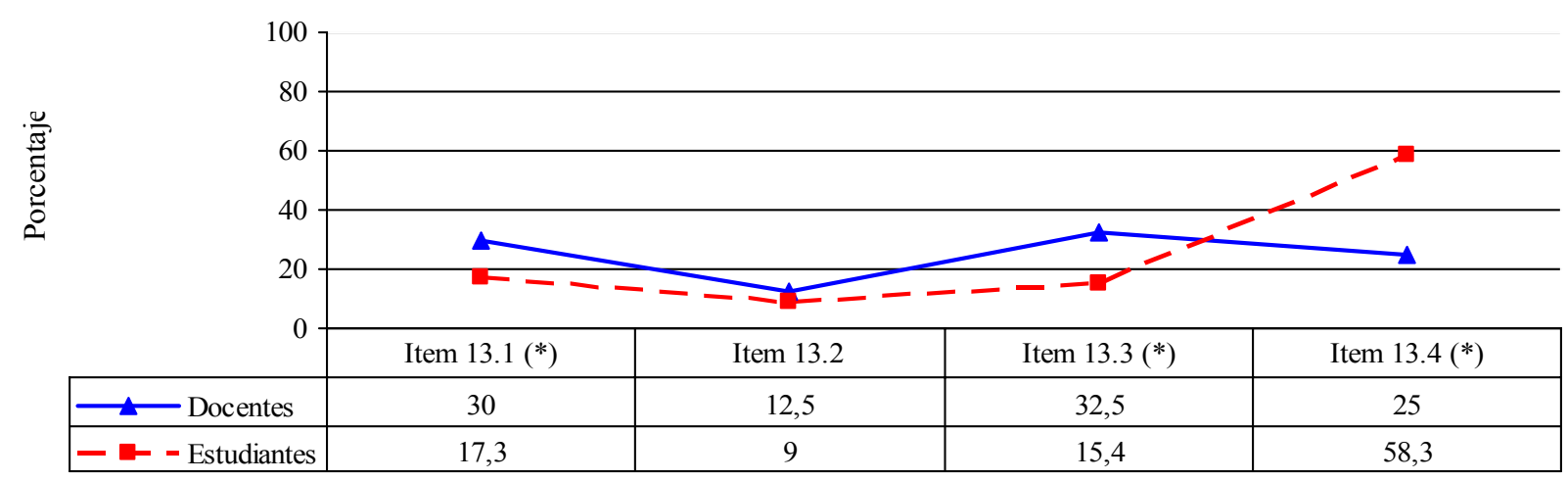

Figura 5. Perspectiva de profesores y estudiantes sobre el uso de la autoevaluación

Existe también cierta divergencia en la respuesta al ítem 13.4, de tal forma que, según la perspectiva del $58,3 \%$ de los estudiantes, el profesorado no pone en práctica estos procesos de autoevaluación, opinión que es sólo compartida por un $25 \%$ del profesorado.
A través de las respuestas al ítem 14 (Figura 6) podemos constatar cómo la autoevaluación se centra en que los docentes proporcionen las soluciones a ejercicios y que sean los propios estudiantes los que los corrijan (ítem 14.2), según la opinión del $30 \%$ del profeso- 
rado y el 33,7\% de los estudiantes. Le sigue la utilización de la autoevaluación como medio a través del cual los estudiantes han de valorar en qué medida cumplen los criterios de evaluación y razonar críticamente esa valoración (ítem 14.5), según el $25 \%$ del profesorado y el $12,9 \%$ de los estudiantes. En menor medida se hace uso de la autoevaluación para identificar y describir errores (ítem 14.4), reflexionar y elaborar un informe sobre lo aprendido (ítem14.3) o autocalificarse (ítem 14.1).

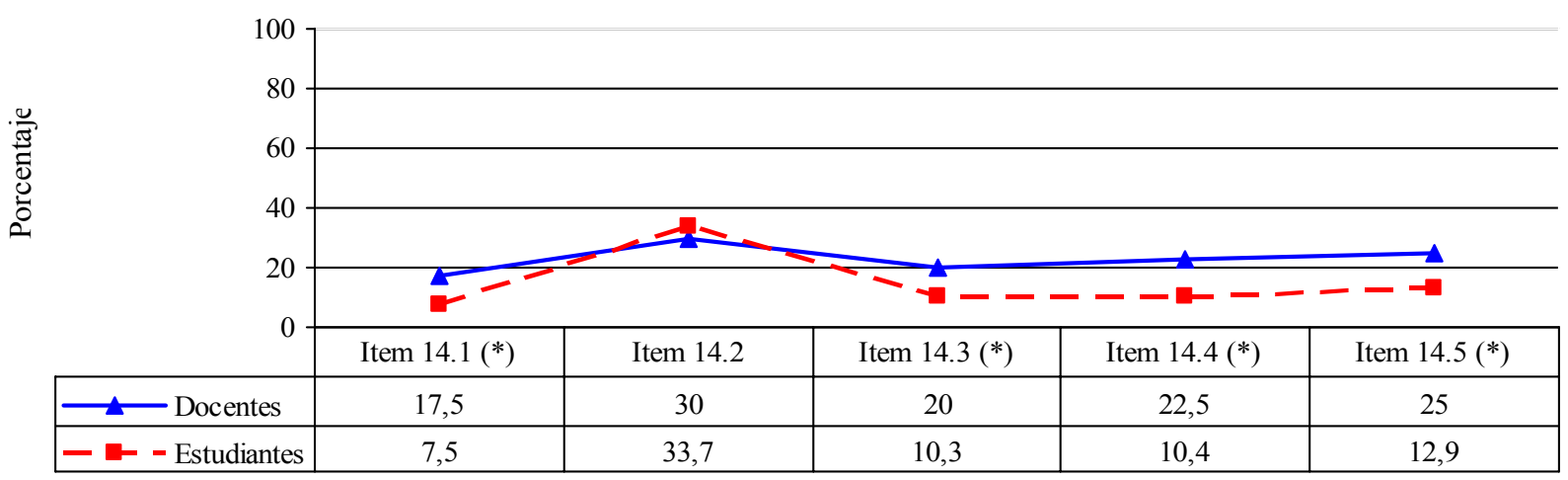

Figura 6. Perspectiva de profesores y estudiantes sobre la forma de la autoevaluación

En segundo lugar, en cuanto a la evaluación entre iguales, la información se refleja en los ítems 15 y 16. En la figura 7 puede observarse la opinión de profesores y estudiantes sobre el uso de esta modalidad de evaluación. Tanto estudiantes $(69,3 \%)$ como docentes $(47,5 \%)$ manifiestan que no se pone en práctica la evaluación entre iguales (ítem
15.4). Cuando se pone en práctica, según la opinión del $22,5 \%$ del profesorado, lo que más se realiza es que los estudiantes valoren las ejecuciones de otros compañeros de su grupo (ítem 15.1); en cambio el $11,5 \%$ de los estudiantes opina que lo que más se pone en práctica es la valoración de las ejecuciones del grupo clase (ítem 15.2).

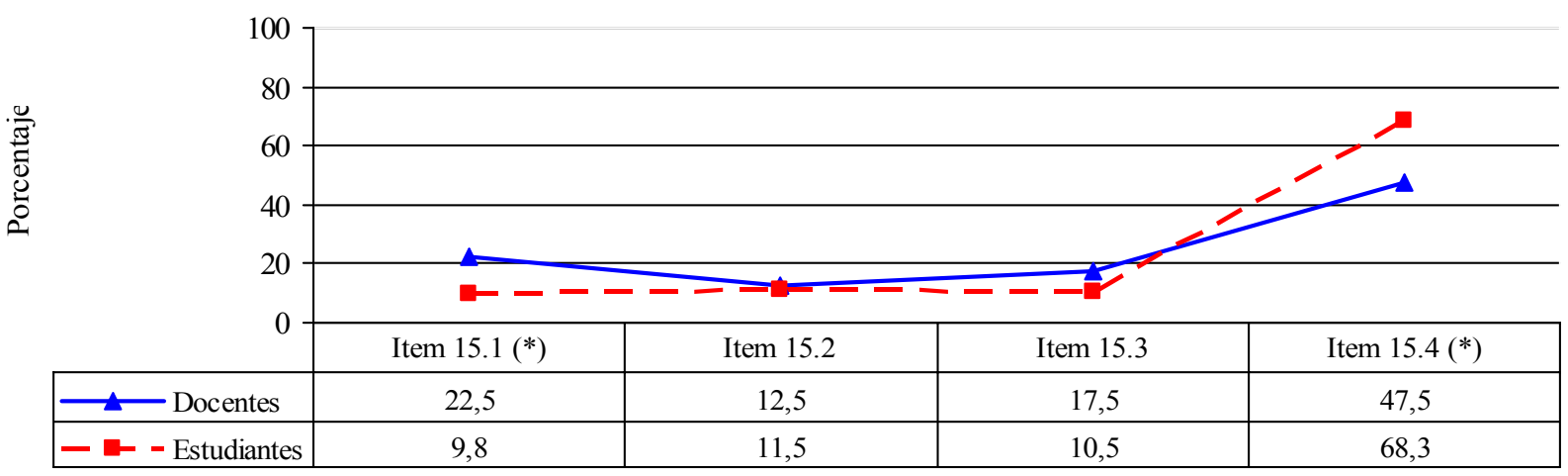

Figura 7. Perspectiva de profesores y estudiantes sobre la utilización de la evaluación entre iguales

En relación a cómo se concreta la evaluación entre iguales (Figura 8), se constata cierta discrepancia entre la percepción de estudiantes y profesorado, manifestada fundamentalmente en los ítems 16.1, $16.2 \mathrm{y}$ 16.4. Mientras el $17,3 \%$ de los estudiantes opina que su participación se centra en corregir los ejercicios de otros compañeros sobre la base de las soluciones aportadas por el profesorado, sólo dice poner en práctica esta posibilidad el 7,5\% del profesorado. Mayor es la discrepancia entre ambas pers- 
pectivas cuando se trata de valorar con criterios de evaluación el trabajo realizado por otros compañeros, razonando críticamente esta valoración. El 27,5\% de los profesores afirma llevarlo a cabo, aspecto que sólo es percibido por un $7,2 \%$ de los estudiantes.

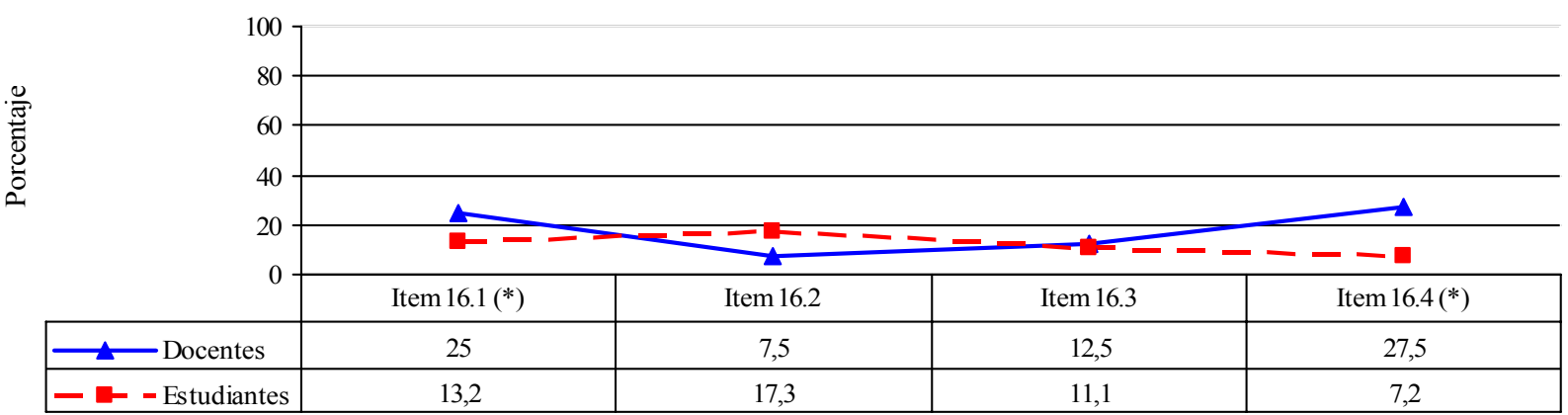

Figura 8. Perspectiva de profesores y estudiantes sobre las formas de la evaluación entre iguales

Por último, sobre la utilización de la coevaluación se preguntó en el ítem 17. Las respuestas obtenidas tanto por los docentes como por los estudiantes desvelan con claridad que, de forma mayoritaria, no se pone en práctica. Tan sólo uno de los profesores encuestados afirma que la desarrolla y, en consonancia con ello, un $92,2 \%$ de los estudiantes declara que el profesorado no practica la coevaluación o evaluación consensuada.

Actuación, beneficios y consecuencias de la participación

La información sobre estos aspectos se recogió a través de los ítems 18, 19 y 20 de la encuesta. En el ítem 18 se recabó la opinión de profesores y estudiantes sobre cómo perciben la actuación de los estudiantes cuando participan en el proceso de evaluación; los ítems 19 y 20 se centraban en las consecuencias y beneficios de dicha participación.

Como puede observarse en la Figura 9, la opinión de docentes y estudiantes sobre la actuación del alumnado en la evaluación mantienen perfiles de respuesta con tendencias muy similares, aunque con diferencias estadísticamente significativas en todos los casos, excepto en el ítem 18.8.

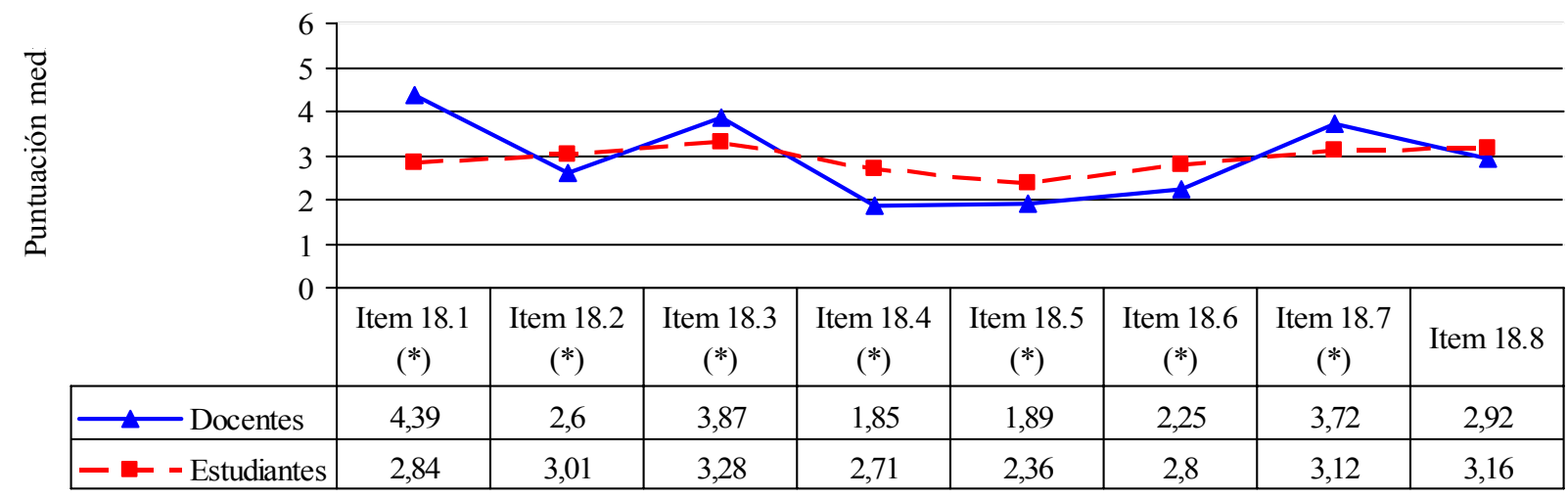

Figura 9. Perspectiva de profesores y estudiantes sobre la actuación de los estudiantes en una evaluación participativa 
Sobre las opiniones de los docentes destaca que la mayoría están de acuerdo en que los estudiantes tienden a sobrevalorar su ejecución (ítem 18.1) y a dar importancia al esfuerzo realizado sin atender a los resultados (ítem 18.3). Además, reconocen que los estudiantes suelen participar en la evaluación cuando se les solicita (ítem 18.7), aunque no están del todo de acuerdo en que los estudiantes tengan buena predisposición inicial para asumir responsabilidades en la propia evaluación o la evaluación de los demás (ítem 18.8).

Asimismo, expresan su desacuerdo con que los estudiantes tengan dominio de la materia para realizar valoraciones objetivas (ítem 18.2), que tengan experiencia para autoevaluarse (ítem 18.4) y para evaluar a sus compañeros (ítem 18.5). Igualmente reconocen que los estudiantes carecen de formación suficiente para realizar la evaluación (ítem 18.6).

Los estudiantes piensan que no tienden a sobrevalorar la ejecución de sus compañeros (ítem 18.1) y coinciden con el profesorado en entender que no tienen un dominio de la materia que les permita realizar valoraciones objetivas (ítem 18.2) y que dan importancia al esfuerzo realizado sin atender a los resultados (ítem 18.3).También manifiestan poca experiencia en la autoevaluación (ítem 18.4) $\mathrm{y}$ en la evaluación entre iguales (ítem 18.5) $\mathrm{y}$, en mayor medida que la opinión del profesorado, consideran no tener suficiente formación para la evaluación (ítem 18.6). Por último, de forma media-baja declaran que suelen participar en la evaluación cuando lo solicita el profesor (ítem 18.7) y que les gusta asumir responsabilidades en su propia evaluación o en la evaluación de los demás (18.8).

Se completó esta información preguntando a ambos colectivos sobre la sinceridad de los estudiantes en la evaluación. Refiriéndonos siempre a las puntuaciones medias, los estudiantes expresan su desacuerdo en relación a que sus valoraciones estén influidas por la posible incidencia que éstas puedan tener en las calificaciones $(2,56)$ o que infravaloren las ejecuciones de sus compañeros $(2,10)$. El profesorado, por su parte, considera que los alumnos son principalmente sinceros en las valoraciones que realizan $(3,38)$. Sin embargo, opinan que las aportaciones de los estudiantes en la discusión o consenso de la calificación tienden a ser subjetivas y sesgadas $(3,79)$.

Por otra parte, con respecto a los beneficios de la participación de los estudiantes en la evaluación, observamos en la Figura 10 que ambos colectivos están de acuerdo en considerar positivos la mayoría de los beneficios asociados a la misma, destacando sobre todo el beneficio del aumento de la capacidad de identificar los propios errores (ítem 20.6) y la mayor implicación en el proceso de aprendizaje (ítem 20.5).

Otros beneficios que destacan tanto el profesorado como los estudiantes, aunque con ligeras variaciones en el orden de importancia, son el desarrollo de una actitud crítica ante las propias realizaciones (ítem 20.2); la mejora de las habilidades y destrezas que se desarrollan en las materias (ítem 20.4); la mejora de los conocimientos propios de la materia (ítem 20.3); la mejora los resultados o productos esperados del aprendizaje (ítem 20.8); la capacidad de mejorar las actitudes (ítem 20.7); y, finalmente, el beneficio que menos valoración obtiene, aunque también con una valoración positiva general en ambos colectivos, es la adquisición de una visión más completa de las competencias a adquirir en la asignatura (ítem 20.1). 


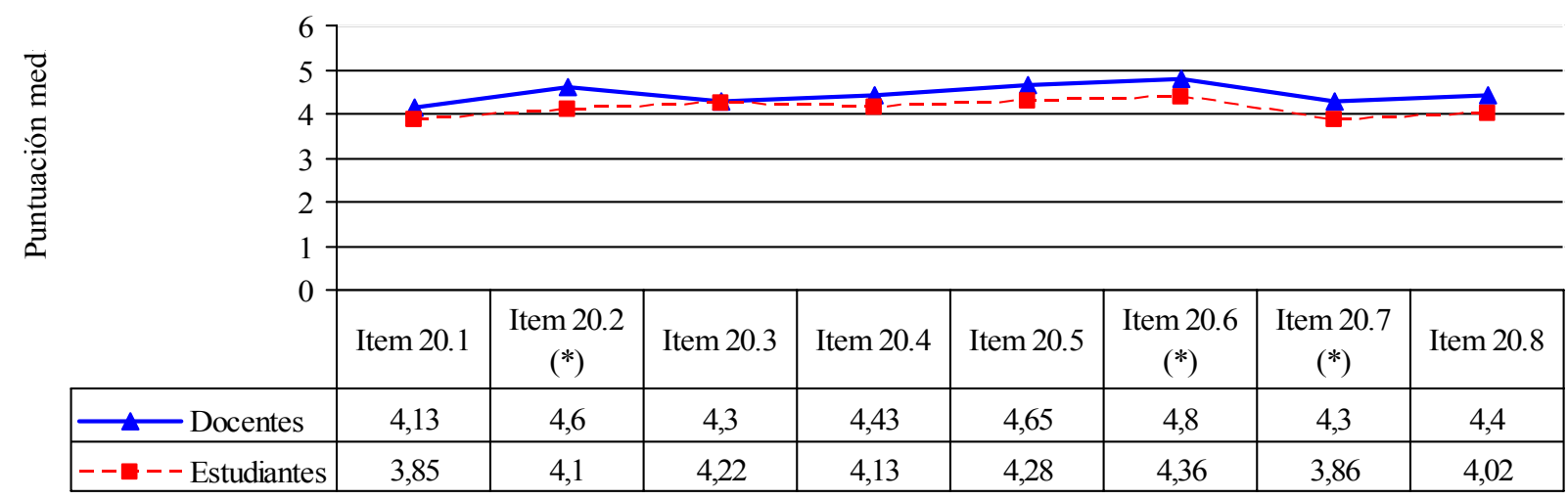

Figura 10. Perspectiva de profesores y estudiantes sobre los beneficios de la participación de los estudiantes en la evaluación

En relación a las posibles consecuencias que podría tener la participación en la evaluación (ver Figura 11), se observan tendencias similares en las respuestas. Ambos colectivos mantienen mayoritariamente $(45,8 \%$ y $41,4 \%$ ) que el profesorado revisa las califi- caciones teniendo en cuenta la autoevaluación (ítem 19.1). En menor medida se produce esta revisión teniendo en cuenta la evaluación entre iguales (ítem 19.2) y en muy pocos casos se lleva a cabo un proceso de consenso en la evaluación (ítem 19.3).

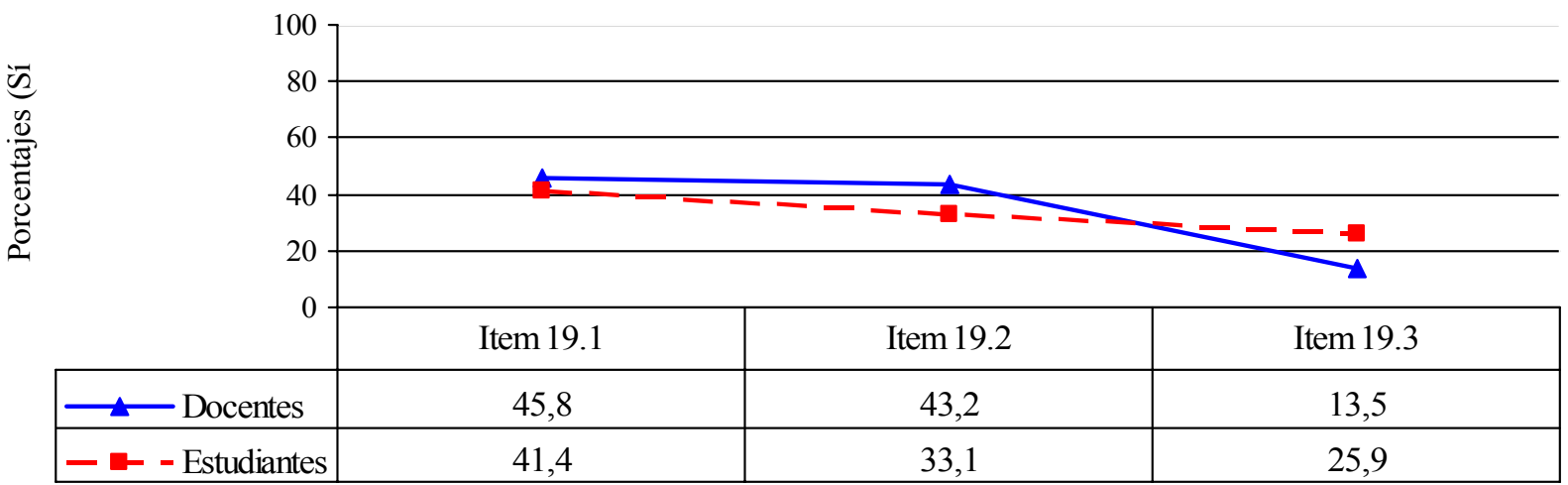

FIGURA 11. Perspectiva de profesores y estudiantes sobre las consecuencias de la participación de los estudiantes en la evaluación

Esta apreciación es coherente con el grado en que profesores y estudiantes manifiestan que se practican estas modalidades de evaluación (ítem 21). Así, podemos observar en la Figura 12 cómo profesores y estudiantes están de acuerdo en que la modalidad mayoritariamente puesta en práctica es la evaluación del profesor, seguida en muy menor medida de las restantes estrategias evaluativas participativas. 


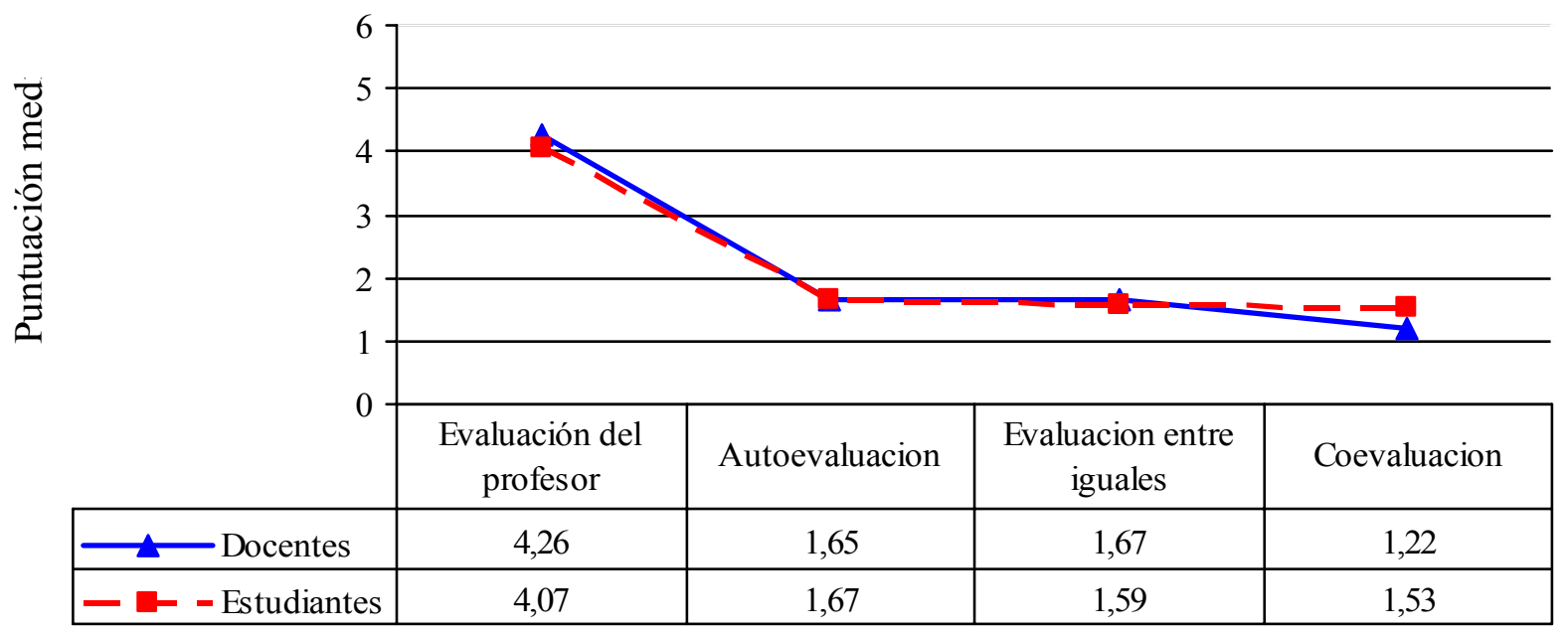

Figura 12. Perspectiva de profesores y estudiantes sobre el grado de utilización de diferentes modalidades de Evaluación

\section{Conclusiones y prospectiva}

Mediante esta investigación se planteó analizar cómo perciben, tanto el profesorado como el alumnado, la participación de los estudiantes universitarios en la evaluación, utilizando como fuente de información los dos colectivos implicados. En primer lugar se analizó la explicitación que los profesores realizan sobre este particular en los programas oficiales de las asignaturas, analizando de forma específica el apartado dedicado a la planificación de la evaluación de sus asignaturas. En segundo lugar, se analizó la opinión que profesores y estudiantes expresaron al responder a dos cuestionarios elaborados al efecto.

Tras el análisis documental de los programas oficiales de las asignaturas podemos concluir que existen escasas evidencias que muestren que el profesorado universitario se plantee como algo importante considerar la participación de los estudiantes en el proceso de evaluación. Esta falta de indicadores sobre la presencia de una evaluación participativa hace que podamos afirmar que el tipo de evaluación que predomina en la institución analizada es el de "evaluación tradicional" ya que sigue siendo un proceso diseñado, ejecutado y controlado esencialmente por parte del docente, no evidenciándose la participación de los estudiantes a través de estrategias evaluativas de carácter alternativo como la autoevaluación, la evaluación entre iguales o la coevaluación.

Desde el análisis descriptivo de la opinión de profesores y estudiantes hemos podido comprobar cómo ambos colectivos muestran en general su acuerdo en los beneficios que la participación activa de los estudiantes en el proceso de evaluación pueden tener en aspectos tales como la adquisición de una visión más completa de las competencias a adquirir en una asignatura, el desarrollo de la capacidad crítica, la implicación activa en el proceso de aprendizaje, la mejora del conocimiento sobre las materias específicas, o la mejora de las actitudes y los resultados o productos de aprendizaje.

Sin embargo este reconocimiento se enfrenta al escaso uso de estrategias participativas en la evaluación. En este sentido, destaca la utilización de la autoevaluación, seguida de la evaluación entre iguales, pero discutir, compartir y consensuar a través de la coevaluación no es una práctica evaluativa que se realice en las aulas universitarias.

Se ha constatado cómo profesores y estudiantes están relativamente de acuerdo en que no está generalizado que profesores y estudiantes colaboren en el diseño de la evaluación a través de, por ejemplo, la selección de tareas 
o instrumentos de evaluación, o para establecer los procedimientos de evaluación.

Por lo que se refiere a los criterios de evaluación, una parte importante del profesorado encuestado se muestra a favor de adoptar alguna estrategia que permita a los estudiantes participar en la determinación de los criterios de evaluación; afirma realizar actividades para explicar y discutir con los estudiantes los criterios de evaluación y modifican los criterios previos incorporando las propuestas de los estudiantes. Estas opiniones son bastante matizadas por los estudiantes quienes, de forma mayoritaria, apuestan por esa necesidad de participación en la evaluación y consideran que la explicación y discusión de los criterios o la participación en la determinación de los mismos no es una realidad generalizada.

Profesores y estudiantes están bastante de acuerdo con que es minoritario el entrenamiento o formación de los estudiantes para poder abordar la evaluación de una manera eficaz. Asimismo están de acuerdo en que el profesorado aporta retroalimentación a sus estudiantes, de tal forma que los estudiantes pueden llegar a conocer su nivel de logro, pero es bastante menor el uso que se hace de la proalimentación, limitando así las posibles mejoras del rendimiento de los estudiantes.

Las conclusiones expuestas deben considerarse desde las limitaciones metodológicas que este estudio tiene, como la reducida muestra utilizada y centrarse en una única institución académica, lo que no permite generalizaciones.

A pesar de estas limitaciones de este estudio descriptivo, los resultados presentados llaman la atención sobre la necesidad de establecer procesos formativos, tanto para el profesorado como para los estudiantes, a través de los cuales se pueda favorecer la participación activa de los estudiantes en los procesos de evaluación y, progresivamente, se vaya considerando la evaluación como un medio para que ambos compartan la responsabilidad del aprendizaje de los estudiantes. En definitiva, es preciso educar en evaluación, de tal forma que pueda hacerse realidad la proposición de Boud et al. (2010) según la cual estudiantes y profesores se convierten en socios responsables del aprendizaje y de la evaluación.

Como nos señala Sadler (2010), la competencia evaluativa es una competencia que ha ido adquiriendo el profesorado, después de enfrentarse a la evaluación de sus estudiantes, mediante el entrenamiento y la práctica y esta misma oportunidad de puesta en práctica es la que deberíamos ofrecer a nuestro alumnado para desarrollar en ellos la capacidad de establecer criterios y valorar de forma crítica y coherente no sólo su aprendizaje sino también el de los demás. En este sentido, los estudiantes también pueden aprender a proporcionar retroalimentación y proalimentación de calidad a sus iguales, lo que contribuiría tanto a mejorar las propias producciones como las de los compañeros, y también a desarrollar competencias de alto nivel como la argumentación o el pensamiento crítico.

El reto de los próximos años radica en ofrecer esta formación específica para los estudiantes, de tal forma que se constituya en una experiencia básica de su paso por la universidad. Pero ello supone y exige que se institucionalice en la universidad la dimensión participativa en los procesos de evaluación.

Falchikov (2005: 254) manifestaba que aún sabemos muy poco sobre muchos aspectos de la implicación de los estudiantes en los procesos de evaluación y reclamaba que sería necesario un programa coordinado de investigación transcultural que permitiera investigar con una mayor profundidad en este campo. El estudio que se ha presentado ha intentado aportar algo de luz en este sentido, pero son muchos los interrogantes que aún quedan abiertos. Desde diferentes proyectos de investigación e innovación ${ }^{[2]}$ se están impulsando estas estrategias evaluativas de carácter participativo y abriendo espacios de comunicación e intercambio entre el profesorado universitario, como se ha podido constatar en las aportaciones realizadas 
en el congreso internacional EVALTrends 2011 (http://evaltrends.uca.es). Confiamos en que en los próximos años se amplíen las investigaciones en este terreno y se generalice el uso de estrategias evaluativas participativas entre el profesorado y los estudiantes universitarios, favoreciendo de esta forma el aprendizaje estratégico a lo largo de la vida.

\section{Referencias}

Biggs, J. (2005). Calidad del aprendizaje universitario. Madrid: Narcea.

Biggs, J. \& Tang, C. (2009). Teaching for Quality Learning at University. Buchingham: Open University Press.

Bordas, M.I. \& Cabrera, F.A. (2001). Estrategias de evaluación de los aprendizajes centrados en el proceso. Revista Española de Pedagogía, LIX (218), 25-48.

Boud, D. (1991). Implementing student selfassessment. Campbelltown: Higher Education Research and Development. Society of Australia Incorporated.

Boud, D. (2000). Sustainable assessment: rethinking assessment for the learning society. Studies in Continuing Education, 22 (2), 151167.

Boud, D. (2006). Foreword. En C. Bryan \& K. Clegg (Eds.), Innovative Assessment in Higher Education (pp. xvii-xix). New York: Routledge.

Boud, D. \& Associates (2010). Assessment 2020: Seven propositions for assessment reform in higher education. Sydney: ALTC.

Brew, A. (2003). La autoevaluación y la evaluación por los compañeros. En S. Brown \& A. Glasner (Eds.), Evaluar en la Universidad. Problemas y nuevos enfoques (pp. 179-189). Madrid: Narcea.

Carless, D., Joughin, G. \& Mok, M. (2006). Learning-oriented assessment: principles and practice, Assessment and Evaluation in Higher Education, 31(4), 395-398.

Dochy, F., Segers, M. \& Sluijsmans, D. (1999). The use of self-, peer and coassessment in higher education: a review. Studies in Higher Education 24 (3), 331-350.
Falchikov, N. (1986). Product comparisons and process benefits of collaborative peer group and self-assessments. Assessment \& Evaluation in Higher Education, 11 (2), 144166.

Falchikov, N. (2005). Improving Assessment Through Student Involvement. Practical solutions for aiding learning in higher and further education. London: Routledge-Falmer.

Gessa Perera, A. (2011). La coevaluación como metodología complementaria de la evaluación del aprendizaje. Análisis y reflexión en las aulas universitarias. Revista de Educación, (354), 749-764. Recuperado de http://www.revistaeducacion.educacion.es/re3 54/re354 30.pdf

Gibbs, G. (1981). Teaching students to learn: a students-centred approach. Philadelphia: Open University Press.

Gibbs, G. (2003). Uso estratégico de la evaluación en el aprendizaje. En S. Brown Y A. Glasner (Eds.), Evaluar en la Universidad. Problemas y nuevos enfoques (pp. 61-76). Madrid: Narcea.

Gibbs, G. (2006). Why assessment is changing. En C. Bryan \& K. Clegg (Eds.), Innovative Assessment in Higher Education (pp. 1122). New York: Routledge.

Gielen, S., Docky, F. \& Onghena, P. (2011). An inventory of peer assessment diversity. Assessment \& Evaluation in Higher Education, 36 (2), 137-155.

Goñi Zabala, J. M. (2005). El espacio europeo de educación superior, un reto para la universidad. Competencias, tareas y evaluación, los ejes del currículum universitario. Barcelona: Octaedro

Ibarra Sáiz, M.S. (1999). Guía para un diagnóstico alternativo en el contexto del aula. Diagnóstico en Educación. Proyecto Docente (pp.167-211). Cádiz: Universidad de Cádiz.

Ibarra Sáiz, M. S. (Dir.) (2007). Proyecto SISTEVAL. Recursos para el establecimiento de un sistema de evaluación del aprendizaje universitario basado en criterios, normas y procedimientos públicos y coherentes. Cádiz: Servicio de Publicaciones de la Universidad 
de Cádiz. Recuperado de http://minerva.uca.es/publicaciones/asp/docs/ obrasDigitalizadas/sisteval/sisteval.html

Ibarra Sáiz, M.S.(Dir.) (2008). EvalCOMIX: Evaluación de competencias en un contexto de aprendizaje mixto. Cádiz: Servicio de Publicaciones de la Universidad de Cádiz. Recuperado el 20 de julio de 2011, de http://minerva.uca.es/publicaciones/asp/docs/ obrasDigitalizadas/evalcomix.pdf

Ibarra Sáiz, M.S. y Rodríguez Gómez, G. (2010). Los procedimientos de evaluación como elementos de desarrollo de la función orientadora en la universidad. Revista Española de Orientación y Psicopedagogía, 21 (2), 443-461.

Ibarra Sáiz, M.S., Rodríguez Gómez, G. y Gómez Ruiz, M.A. (2012). La evaluación entre iguales: beneficios y estrategias para su práctica en la universidad. Revista de Educación, (359). Avance de publicación on-line. DOI: 10-4438/1988-592X-RE-2010-359-092

Knight, P. T. (2005). El profesorado de Educación Superior. Formación para la Excelencia. Madrid: Narcea.

López Pastor, V.M. (Coord.) (2009). La evaluación formativa y compartida en docencia universitaria: propuestas, técnicas, instrumentos y experiencias. Madrid: Narcea.

Ljungman, A. \& Silén, C. (2008). Examination involving students as peer examiners. Assessment \& Evaluation in Higher Education, 33 (3), 289-300.

Padilla Carmona, M.T. y Gil Flores, J. (2008). La evaluación orientada al aprendizaje en la Educación Superior: Condiciones y estrategias para su aplicación en la docencia universitaria. Revista Española de Pedagogía, 66 (24) 467-486.

Pérez Pueyo, A., Tabernero B., López, V.M., Ureña, N., Ruiz, E., Caplloch, M., González, N. y Castejón, F.J. (2008). Evaluación formativa y compartida en la docencia universitaria y el Espacio Europeo de Educación Superior: cuestiones clave para su puesta en práctica. Revista de Educación, (347), 435-451. Recuperado

de http://www.revistaeducacion.mec.es/re347/re347 20.pdf

Prins, F.J., Sluijmans, M.A., Kirschener, P.A. y Strijbos, J. W. (2005). Formative peer assessment in a CSCL environment: a case study. Assessment \& Evaluation in Higher Education, 30(4), 417-444.

Nicol, D. (2009). Transforming Assessment and Feedback: Enhancing integration and empowerment in the first year. Mansfield: Enhacement Themes.

Rodríguez Gómez, G., Gil Flores, J. y García Jiménez, E. (1999). Metodología de la investigación cualitativa. Archidona, MA: Aljibe.

Rodríguez Gómez, G. (Dir.) (2009). EvalHIDA: Evaluación de Competencias con Herramientas de Interacción Dialógica Asíncronas (foros, blogs y wikis). Cádiz: Servicio de Publicaciones de la Universidad de Cádiz. Recuperado el 20 de julio de 2011, de http://www.tecn.upf.es/ daviniah/evalhida.pdf

Rodríguez Gómez, G. e Ibarra Sáiz, M.S. (Eds.) (2011). e-Evaluación orientada al eAprendizaje estratégico en la educación superior. Madrid: Narcea.

Rodríguez Gómez, G., Ibarra Sáiz, M.S. y Gómez Ruiz, M.A. (2011). e-Autoevaluación en la universidad: un reto para profesores y estudiantes. Revista de Educación. Avance de publicación on-line. DOI:10-4438/1988592X-RE-2010-356-045.

Rodríguez Gómez, G. (Dir.), Quesada Serra, V., Gómez Ruiz, M. A., Ibarra Sáiz, Ma S., Gallego Noche, B., Cabeza Sánchez, D., León Rodríguez, A., Cubero Ibáñez, J. (2010). ReEvalúa: Comprobando el impacto de la eEvaluación orientada al e-Aprendizaje en la universidad. En M.E. Prieto Méndez, J.M. Dodero Beardo y D.O. Villegas Sáenz (Eds.), Recursos Digitales para la Educación y la Cultura. Actas CcITA-Volumen SPDECE (pp. 253-256). Cádiz: Universidad de Cádiz y Universidad Tecnológica Metropolitana de México.

Sadler, D. R. (2010). Beyond feedback: Developing student capability in complex appraisal. Assessment and Evaluation in Higher Education, 35, 535-550. 
Sambell, K. y McDowell, L. (1998). The construction of the hidden curriculum: messages and meanings in the assessment of student learning. Assessment and Evaluation in Higher Education, 23, 391-402.
Sivan, A. (2000). The implementation of peer assessment: an action research approach. Assessment in Education, 7(2), 193-213.

Stefani, L.A.J. (1994). Peer, self and tutor assessment: relative reliabilities. Assessment and Evaluation in Higher Education, 19(1), 69-75.

\section{NOTAS}

${ }^{\text {[1] }}$ Proyecto EvalPART - La participación de los estudiantes en la evaluación del aprendizaje y calidad en la Educación Superior, financiado por la Agencia Española de Cooperación Internacional para el Desarrollo (AECID) (Ref. A/016477/08).

${ }^{\text {[2] }}$ Proyecto de excelencia Re-Evalúa -"Reingeniería de la e-evaluación, tecnologías y desarrollo de competencias en profesores y estudiantes universitarios". Ref. P08-SEJ-03502. http://reevalua.uca.es. Proyecto INEVALCO - "Innovación en la Evaluación de Competencias. Diseño y desarrollo de procedimientos e instrumentos para la evaluación de competencias en entornos de aprendizaje mixtos/virtuales con la participación de los estudiantes en los títulos de grado". Ref. EA-2010-0052. 


\section{ABOUT THE AUTHORS / SOBRE LOS AUTORES}

Rodríguez-Gómez, Gregorio (gregorio.rodriguez@uca.es). Profesor titular del área de 'Métodos de Investigación y Diagnóstico en Educación' (MIDE) de la Universidad de Cádiz (España). Es el autor de contacto para este artículo. Sus principales ámbitos de investigación son la metodología cualitativa y la evaluación educativa. Fue el fundador y primer Director Ejecutivo de RELIEVE, desde 1994 hasta el año 2001. Su dirección postal es: Facultad de Ciencias de la Educación. Campus Universitario de Puerto Real. 11510 - Puerto Real, Cádiz (España). Buscar otros artículos de este autor en Google Académico / Find other articles by this author in Scho$\underline{\text { lar Google }}$

Ibarra-Sáiz, Ma Soledad (marisol.ibarra@uca.es). Profesora titular del área de 'Métodos de Investigación y Diagnóstico en Educación' (MIDE) de la Universidad de Cádiz (España). Dirige el grupo de investigación EVALfor. Su principal ámbito de investigación es la evaluación educativa. Su dirección postal es: Facultad de Ciencias de la Educación. Campus Universitario de Puerto Real. 11510 - Puerto Real, Cádiz (España). Buscar otros artículos de esta autora en Google Académico / Find other articles by this author in Scholar Google

\section{Google}

Gallego Noche, Beatriz (beatriz.gallego@uca.es). Profesora Contratada Doctora del área de Métodos de Investigación y Diagnóstico en Educación (MIDE) de la Facultad de Ciencias de la Educación de la Universidad de Cádiz. Miembro del grupo de investigación EVALfor. Sus principales ámbitos de investigación son la evaluación educativa y los estudios de género. Su dirección postal es: Facultad de Ciencias de la Educación. Campus Universitario de Puerto Real. 11510 - Puerto Real, Cádiz (España).

Gómez-Ruiz, Miguel-Ángel (miguel.gomez@uca.es) Profesor del área de 'Métodos de Investigación y Diagnóstico en Educación (MIDE) de la Facultad de Ciencias de la Educación de la Universidad de Cádiz (España). Miembro del grupo de investigación EVALfor. Su dirección postal es: Facultad de Ciencias de la Educación. Campus Universitario de Puerto Real. 11510 - Puerto Real, Cádiz (España).

Quesada Serra, Victoria (victoria.quesada@uca.es). Profesora del área de Métodos de Investigación y Diagnóstico en Educación de la Facultad de Ciencias de a Educación de la Universidad de Cádiz. Miembro del grupo de investigación EVALfor. Su principal ámbito de investigación es la evaluación educativa. Su dirección postal es: Facultad de Ciencias de la Educación. Campus Universitario de Puerto Real. 11510 - Puerto Real, Cádiz (España). 
Rodríguez-Gómez, Gregorio; Ibarra, Marisol; Gallego-Noche, Beatriz; Gómez-Ruiz, Miguel-Ángel \& Quesada-Serra, Victoria (2012). La voz del estudiante en la evaluación del aprendizaje: un camino por recorrer en la universidad.

RELIEVE, v. 18, n. 2, art. 2. DOI: 10.7203/relieve.18.2.1985

\title{
ARTICLE RECORD / FICHA DEL ARTÍCULO
}

\begin{tabular}{|c|c|}
\hline $\begin{array}{l}\text { Reference / } \\
\text { Referencia }\end{array}$ & 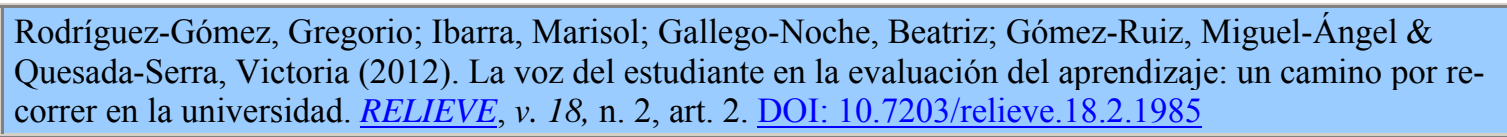 \\
\hline Title / Título & $\begin{array}{l}\text { La voz del estudiante en la evaluación del aprendizaje: un camino por recorrer en la universidad. [Student } \\
\text { voice in learning assessment: a pathway not yet developed at university]. }\end{array}$ \\
\hline $\begin{array}{l}\text { Authors / } \\
\text { Autores }\end{array}$ & $\begin{array}{l}\text { Rodríguez-Gómez, Gregorio; Ibarra-Sáiz, Maria Soledad; Gallego-Noche, Beatriz; Gómez-Ruiz, Miguel- } \\
\text { Ángel \& Quesada-Serra, Victoria }\end{array}$ \\
\hline $\begin{array}{l}\text { Review / } \\
\text { Revista }\end{array}$ & RELIEVE (Revista ELectrónica de Investigación y EValuación Educativa), v. 18, n. 2 \\
\hline ISSN & $1134-4032$ \\
\hline $\begin{array}{l}\text { Publication } \\
\text { date / } \\
\text { Fecha de } \\
\text { publicación }\end{array}$ & $\begin{array}{l}2012 \text { (Reception Date: } 2011 \text { June } 08 \text {; Approval Date: } 2012 \text { August 30. Publication Date: } 2012 \text { Septem- } \\
\text { ber 03). }\end{array}$ \\
\hline $\begin{array}{l}\text { Abstract / } \\
\text { Resumen }\end{array}$ & $\begin{array}{l}\text { During 2009/2010, the current research was conducted with the aim of analysing student and teaching } \\
\text { staff on regarding student participation in assessment. A content analysis of } 76 \text { subject outlines was car- } \\
\text { ried out, and then } 40 \text { member of the teaching staff and } 614 \text { university students were surveyed. The results } \\
\text { of the content analysis prove there is a shortage of information about and programming for student partic- } \\
\text { ipation in assessment. } \\
\text { Durante el curso } 2009 / 2010 \text { se llevó a cabo esta investigación con el objetivo de analizar la opinión y pers- } \\
\text { pectiva que profesores y estudiantes universitarios tienen sobre la participación de estos últimos en el pro- } \\
\text { ceso de evaluación. Se realizó un análisis de contenido de } 76 \text { programas de asignaturas universitarias y se } \\
\text { encuestaron mediante dos cuestionarios a } 40 \text { profesores y } 614 \text { estudiantes universitarios. Los resultados } \\
\text { muestran una escasez de evidencias sobre la participación real de los estudiantes. Además, confirman opi- } \\
\text { niones divergentes entre docentes y estudiantes sobre los usos y las formas en las que se concreta esta par- } \\
\text { ticipación activa en la evaluación. }\end{array}$ \\
\hline \multirow{2}{*}{$\begin{array}{l}\text { Keywords / } \\
\text { Descriptores }\end{array}$} & $\begin{array}{l}\text { Learning assessment, Learning-oriented assessment, Self-assessment, Peer-assessment, Co-assessment, } \\
\text { Collaborative assessment, Participative assessment, Higher Education. }\end{array}$ \\
\hline & $\begin{array}{l}\text { Evaluación del aprendizaje, evaluación orientada al aprendizaje, autoevaluación, evaluación entre iguales, } \\
\text { coevaluación, evaluación colaborativa, evaluación participativa, educación superior. }\end{array}$ \\
\hline $\begin{array}{l}\text { Institution / } \\
\text { Institución }\end{array}$ & Facultad de Educación. Universidad de Cádiz (España). \\
\hline $\begin{array}{l}\text { Publication site } \\
\text { / Dirección }\end{array}$ & http://www.uv.es/RELIEVE \\
\hline $\begin{array}{l}\text { Language / } \\
\text { Idioma }\end{array}$ & Español \& English version (Title, abstract and keywords in English \& Spanish) \\
\hline
\end{tabular}

\section{RELIEVE}

\section{Revista ELectrónica de Investigación y $\mathbf{E V}$ aluación Educativa E-Journal of Educational Research, Assessment and Evaluation}

[ISSN: 1134-4032]

\begin{abstract}
(C) Copyright, RELIEVE. Reproduction and distribution of this articles it is authorized if the content is no modified and their origin is indicated (RELIEVE Journal, volume, number and electronic address of the document).

(C) Copyright, RELIEVE. Se autoriza la reproducción y distribución de este artículo siempre que no se modifique el contenido y se indique su origen (RELIEVE, volumen, número y dirección electrónica del documento).
\end{abstract}

